\title{
Persistent N-cadherin Expression Induced by Extended Epidermal Growth Factor Exposure Regulates Multicellular Aggregate Compaction and Sensitivity to Cisplatin
}

\section{Sabrina L Samudio-Ruiz* and Laurie G Hudson}

Department of Pharmaceutical Sciences, College of Pharmacy, University of New Mexico, Albuquerque, NM, USA

\begin{abstract}
Objective: In ovarian cancer, activation of the epidermal growth factor receptor (EGFR) is associated with poor prognosis. The presence of EGFR activators in patient ascites fluid may cause constitutive EGFR activation thereby contributing to metastasis and/or resistance to therapy. Our goal was to identify alterations resulting from constitutive EGFR activation that influence cell behavior and drug sensitivity.

Methods: We used an in vitro model (OVCA 433 cells) to evaluate changes in mesenchymal marker levels and multicellular aggregate (MCA) formation following long term epidermal growth factor (EGF) treatment. We determined sensitivity to cisplatin following EGF treatment and evaluated the role of the mesenchymal marker, $\mathrm{N}$-cadherin, in aggregate formation and sensitivity using siRNA.

Results: We found that EGFR activation led to phenotypic and functional changes in ovarian tumor cells that were retained after ligand was withdrawn (removal). Expression levels of the mesenchymal markers $\mathrm{N}$-cadherin and vimentin were elevated in EGF treated and removal cells. This persistent increase in mesenchymal markers was associated with a significant increase in (MCA) compaction and cell spreading when MCAs were plated on collagen. $\mathrm{N}$-cadherin silencing decreased MCA compaction and spreading in cells following extended exposure to EGF or in cells with high endogenous levels of $\mathrm{N}$-cadherin. Furthermore, the compact MCA structure in EGF treated cells with increased $\mathrm{N}$-cadherin expression conferred resistance to cisplatin and $\mathrm{N}$-cadherin silencing largely restored cisplatin sensitivity.

Conclusion: Our results indicate that prolonged EGFR activation causes a persistent change in mesenchymal marker expression, which regulates compaction and drug sensitivity. The findings implicate N-cadherin as a key regulator of the EGFR-dependent functional changes in MCAs such as compaction, spreading and sensitivity to platinum-based chemotherapeutics. These findings suggest a mechanism by which persistent EGFR activation in the microenvironment may drive changes in ovarian cancer cells that contribute to the poor prognosis associated with EGFR activation.
\end{abstract}

Keywords: Ovarian cancer; Epidermal Growth Factor Receptor (EGFR); E-cadherin; N-cadherin; Multicellular aggregates; Cisplatin resistance

Abbreviations: DNMT: DNA methyltransferase; EGFR: Epidermal Growth Factor Receptor; EGF: Epidermal Growth Factor; EOC: Epithelial ovarian cancer; EMT: Epithelial to Mesenchymal Transition; ECM: Extracellular Matrix; HB-EGF: Heparin-binding EGF; LPA: Lysohposphatidic Acid; MCA: Multicellular Aggregate; OVCA: Ovarian Cancer

\section{Introduction}

Accounting for approximately $6 \%$ of all cancer related deaths in U.S. women, ovarian cancer remains the most lethal of all gynecological malignancies [1]. Dissemination of the disease beyond the ovaries and late stage diagnosis are major factors in the low 5-year survival rate for epithelial ovarian cancer (EOC), 44\% [1,2]. EOC metastasis occurs when tumor cells shed from the primary tumor into the peritoneal cavity where they can exist as free floating cells or multicellular aggregates (MCA) [3,4]. MCAs, sometimes referred to as spheroids, are normally occurring in ovarian cancer patients, have been isolated from patient ascites and can range in size from 30 to $750 \mu \mathrm{m}$ [5-7]. They are said to be key components of metastasis as they are known to adhere to, disaggregate and infiltrate mesothelial monolayers or extracellular matrix (ECM) substrates like collagen $[5,8]$. Recent studies showing MCAs forcing mesothelial cells aside are further evidence that MCAs play a major role in metastasis [4]. Ovarian cancer MCAs have reduced proliferation and arrest in $\mathrm{G}_{0}$; however, they reattach to adherent surfaces rapidly and retain their ability to re-enter the cell cycle after reattachment occurs [2]. MCAs may therefore play a role in peritoneal dissemination as their quiescent characteristics make them resistant to chemotherapy; thus, representing a chemoresistant population of cells that can contribute to recurrence $[2,8]$.

Ovarian cancer patient ascites contains activators of the Epidermal Growth Factor Receptor (EGFR), such as heparin-binding EGF (HBEGF) and lysophosphatidic acid (LPA), thereby creating a tumor microenvironment that may cause constitutive EGFR activation [911]. The EGFR, and other members of the EGFR family, are known to regulate many different cellular processes (for review see $[12,13]$ ). Within the ovary, EGFR activation regulates growth of the ovarian follicle and postovulatory repair, but it also plays a role in ovarian cancer metastasis $[10,14]$. Activation of the EGFR in patient tumors is associated with increased malignancy and poor patient outcome [14].

Activation of the EGFR is also known to promote epithelial to

*Corresponding author: Sabrina Samudio-Ruiz, Ph.D. University of New Mexico MSC09 5360, Albuquerque, NM 87120, USA, Tel: (505) 272-6932; Fax: (505) 272 0704; E-mail: SRuiz@salud.unm.edu

Received March 19, 2014; Accepted April 14, 2014; Published April 16, 2014

Citation: Samudio-Ruiz SL, Hudson LG (2014) Persistent N-cadherin Expression Induced by Extended Epidermal Growth Factor Exposure Regulates Multicellular Aggregate Compaction and Sensitivity to Cisplatin. Med chem S1: 005 doi:10.4172/2161-0444.S1-005

Copyright: (C) 2014 Samudio-Ruiz SL, et al. This is an open-access article distributed under the terms of the Creative Commons Attribution License, which permits unrestricted use, distribution, and reproduction in any medium, provided the original author and source are credited. 
mesenchymal transition (EMT) $[3,15,16]$. EMT refers to a process by which cells lose the cell-cell contacts and become migratory [17]. Classical characteristics of EMT include 1) loss of epithelial markers such as E-cadherin mediated by epigenetic silencing or induction of transcription factors such as Snail, Slug and Twist as well as 2) induction of mesenchymal markers such as $\mathrm{N}$-cadherin and vimentin $[3,17]$. This change in cadherin expression is often termed "cadherin switching" which can refer to a shift from E-cadherin to N-cadherin expression or expression of $\mathrm{N}$-cadherin or other cadherins without changes in E-cadherin levels [17]. Cadherin switching and EMT, while important in development and wound healing, are also implicated in tumor progression and metastasis; thus, the presence of EMT markers correlates with poor patient prognosis [18-21].

Mesenchymal markers like $\mathrm{N}$-cadherin and vimentin have been implicated in MCA formation and compaction [8]. Studies evaluating spheroid compaction have shown that compact MCAs are more resistant to chemotherapeutics [5]. This work aimed to evaluate the role of EGFR activation on mesenchymal marker induction, spheroid compaction and subsequent response to chemotherapeutics. We hypothesized that mimicking the tumor microenvironment of constitutive EGFR activation would result in EMT and mesenchymal marker induction as well as more compact MCAs. We found that not only did EGFR activation result in the acquisition of EMT characteristics, increased expression of mesenchymal markers and compact MCAs, but that the phenotype of compact MCAs as well as mesenchymal marker induction was a persistent phenotype following prolonged EGFR activation. Furthermore, we also observed that MCA compaction resulted in increased resistance to cisplatin and this compaction is, in part, dependent on expression of N-cadherin. In all, these experiments indicate that activation of the EGFR in the ovarian tumor microenvironment may lead to unfavorable changes in tumor cell behavior, MCA compaction and response to chemotherapeutics.

\section{Materials and Methods}

\section{Cell culture}

The ovarian carcinoma cell line OVCA 433 was provided by Dr. Robert Bast Jr., M.D. Anderson Cancer Center, Houston TX and grown in Minimum Essential Medium Eagle (MEME) (Sigma, St. Louis, MO) supplemented with $10 \%(\mathrm{v} / \mathrm{v})$ fetal bovine serum (FBS) (Gibco, Life Technologies, Carlsbad, CA), $1 \mathrm{mM}$ sodium pyruvate (Sigma), $2 \mathrm{mM} \mathrm{L}$-glutamine (Sigma), 0.5 units/mL penicillin (Sigma), $0.5 \mu \mathrm{g} /$ $\mathrm{mL}$ streptomycin (Sigma), later referred to as MEME growth media. SKOV3 cells purchased from ATCC were grown in RPMI 1640 Media (Sigma) supplemented as described for MEME media shown above. All cells were maintained at $37^{\circ} \mathrm{C}$ under $5 \% \mathrm{CO}_{2} / 95 \%$ air. Long term EGFR activation in the OVCA 433 cells was conducted for at least 40 days using $10 \mathrm{nM}$ EGF (Biomedical Technologies, Stoughton, MA) added to MEME growth media and media -/+ EGF was changed every other day. Following the long term EGF paradigm for at least 40 days, EGF was removed and cells were kept in culture for at least 7 additional days in MEME growth media. This was done in order to observe cellular response to a withdrawal of EGF; these samples were termed removal samples. All long term EGF data was evaluated against an untreated passage control cells to eliminate any confounding variables associated with long cell passage and none of the cells were used passed passage 140. Data from long term EGF treated cells were collected from 4 independent treatment trials to eliminate confounding variables associated with colony selection in a single trial.

\section{Immunoblotting}

Cells were washed with PBS and harvested in cell lysis buffer containing $5 \mathrm{mM}$ EDTA, $2 \mathrm{mM}$ EGTA, $1 \mathrm{mM} \mathrm{Na} \mathrm{VO}_{4}$, leupeptin 10 $\mathrm{mg} / \mathrm{ml}$, pepstatin A $10 \mathrm{mg} / \mathrm{ml}, 1 \mathrm{mM}$ PMSF in PBS and $1 \%$ SDS. Total protein concentrations were determined using the BCA protein assay kit (Thermo Fisher Scientific, Rockford, IL). Equal amounts of total cell lysates $(30 \mu \mathrm{g})$ were electrophoresed through $10 \%$ SDS-polyacrylamide, transferred to $0.45 \mu \mathrm{m}$ nitrocellulose (Biorad, Hercules, CA) and blocked with $3 \%$ BSA. Blots were probed with anti-E-cadherin antibody (Dako, Carpinteria, CA), anti-N-cadherin antibody (BD Transduction Laboratories, Franklin Lakes, NJ), anti-vimentin antibody (Millipore, Chemicon, Billerica, MA) and an anti-GAPDH antibody (Millipore), which was used as a loading control. Blots were then incubated in the appropriate secondary antibody (Promega, Madison, WI) and the immunoreactive proteins were detected using SuperSignal West Pico or Femto Chemiluminescence (Thermo Fisher Scientific). Imaging of the blots and densitometry was accomplished using the Kodak Image Station 440 and related software (NEN Life Science Products, Boston, MA).

\section{RNA isolation and Quantitative Real Time PCR (Q-PCR)}

RNA was extracted from cells using a Trizol reagent (Invitrogen) as recommended by the vendor. cDNA was synthesized using the High Capacity cDNA Reverse Transcription kit (Applied Biosystems, Life Technologies, Grand Island, NY) according to the manufacture's protocol. Briefly, a $2 \mathrm{X}$ reverse transcriptase (RT) master mix containing dNTPs, random primers, RT buffer and MultiScribe Reverse Transcriptase was added to $2 \mu \mathrm{g}$ of RNA and brought up to $20 \mu$ with RNase free $\mathrm{dH}_{2} 0$. Each sample was then put into the thermal cycler and incubated for 10 minutes at $25^{\circ} \mathrm{C}$, followed by 2 hours at $37^{\circ} \mathrm{C}$ and held at $4^{\circ} \mathrm{C}$ overnight. cDNA products were then diluted 1:5 and $2 \mu \mathrm{l}$ of diluted cDNA was used for quantitative real-time PCR (Q-PCR). Q-PCR reactions were performed in a 384-well plate using Syber Green PCR master mix (Applied Biosystems) and primers with a final concentration of $0.2 \mu \mathrm{M}$. E-cadherin (CDH1), N-cadherin (CDH2), vimentin and GAPDH Quantitect primers (Qiagen) were used. Thermal cycling was done in a 7900 HT Fast Real Time PCR system (Applied Biosystems) and started with an initial denaturing at $95^{\circ} \mathrm{C}$ for $10 \mathrm{~min}$, followed by 40 cycles of denaturing at $95^{\circ} \mathrm{C}$ for $30 \mathrm{~s}$, annealing at $56^{\circ} \mathrm{C}$ for $40 \mathrm{~s}$, and extension at $72^{\circ} \mathrm{C}$ for $30 \mathrm{~s}$. The relative quantification of the target gene was determined using the $2^{-\Delta \Delta C t}$ method described by [22]. Briefly, the $\Delta \mathrm{Ct}$ value for each sample was determined as the difference between then average of the duplicate $\mathrm{Ct}$ values for the target gene and the internal control GAPDH $\left(\Delta \mathrm{Ct}_{\text {sample }}\right.$ $\left.={ }^{\text {ave }} \mathrm{Ct}_{\text {gene }}{ }^{-\mathrm{ave}} \mathrm{Ct}_{\mathrm{GAPDH}}\right)$. Then the $\Delta \mathrm{Ct}$ values for all control, untreated OVCÅ 433 cells were averaged (ave $\Delta \mathrm{Ct}_{\text {controls }}$ ) and subtracted from the $\Delta \mathrm{Ct}$ value for each sample which gives the $\Delta \Delta \mathrm{Ct}$ for each sample $\left(\Delta \mathrm{Ct}_{\text {sample }}{ }^{- \text {ave }} \Delta \mathrm{Ct}_{\text {controls }}=\Delta \Delta \mathrm{Ct}_{\text {sample }}\right)$. This was, in turn, used to calculate the $2^{-\Delta \Delta \mathrm{Ct}}$ for each sample. Because $2^{\circ}$ is one, the mean $2^{-\Delta \Delta \mathrm{Ct}}$ value for controls was approximately equal to one, depending on the variability within the control samples. Thus, the experimental sample values were expressed relative to one. All samples were run in duplicate and data is representative of 5 independent experiments.

\section{Multicellular aggregate (MCA) formation and spreading}

SKOV3 and OVCA 433 [control, long term EGF treated and cells removed from EGF for at least 7 days (removal)] were trypsinized, counted and 2500 cells per well were replated in 96 well Lipidure U bottom plates (NOF America Corporation, White Plains, NY). Cells were allowed to aggregate overnight at $37^{\circ} \mathrm{C}$ under $5 \% \mathrm{CO}_{2} / 95 \%$ air. Following MCA formation, MCAs were transferred to collagen type I coated plates using sterile transfer pipettes and allowed to spread overnight at $37^{\circ} \mathrm{C}$ under $5 \% \mathrm{CO}_{2} / 95 \%$ air. Images of MCA formation and spreading were taken using Olympus IX70 equipped with DP72 
digital camera and imaging software. Formed MCAs and spreading areas for each group were determined using the associated Olympus imaging software. Briefly, using the closed polygon tool (Olympus imaging software), the area of either the MCA or the cell spreading area was traced in its entirety and enclosed into a closed polygon. The corresponding area of the polygon was calculated by the imaging software using the information obtained from image acquisition. MCAs and cell spreading experiments were done in duplicate and the corresponding area is an average of those duplicates taken from 4-6 independent experiments.

\section{siRNA}

siRNA duplexes for $\mathrm{CDH} 2$ (N-cadherin) and scramble siRNA were purchased from Origene (Rockville, MD) and reconstituted according to manufacturer's protocol. Silencing of $\mathrm{N}$-cadherin was carried out using HiPerFect HTS transfection reagent (Qiagen, Germantown, $\mathrm{MD)}$ according to manufacturer's protocol. Briefly, $6 \times 10^{4}$ cells per well of either SKOV 3 or long term EGF treated OVCA 433 cells were seeded into 24 well plates. $75 \mathrm{ng}$ of siRNA duplexes and scramble siRNA were preincubated with $3 \mu \mathrm{l}$ of HiPerFect HTS reagent to allow formation of transfection duplexes and then added to the cells. Cells were allowed to incubate with siRNA transfection duplexes at $37^{\circ} \mathrm{C}$ under $5 \% \mathrm{CO}_{2} / 95 \%$ air for 24 hours. Following incubation with siRNA, cells were either collected for protein isolation and immunoblotting or were further split for MCA formation and spreading analysis as described above. Preliminary experiments showed that siRNA mediated knockdown of N-cadherin for 24 hours resulted in decreased protein levels for approximately 4 days (data not shown).

\section{Cisplatin treatment and cell viability}

Monolayers of cells, MCAs and cells treated with either $\mathrm{N}$-cadherin siRNA or scramble siRNA then allowed to form MCAs were also treated with increasing doses of cisplatin (Sigma) $[0-300 \mu \mathrm{M}]$ for 48 hours. Cell viability was assayed using PrestoBlue ${ }^{\circledast}$ (Life Technologies) according to manufacturer's protocol. Briefly, $10 \mu \mathrm{l}$ of PrestoBlue $\mathrm{e}^{\circ}$ reagent was added per every $100 \mu \mathrm{l}$ of media and incubated for 1 hour (monolayer) or 20 hours (MCAs). Preliminary experiments showed that longer incubations for MCAs achieved more consistent results without reaching saturation perhaps due to low cell number and decreased proliferation in this type of 3D cellular model, while 1 hour incubation was sufficient to achieve consistent results and avoid saturation for the monolayer model. After the respective incubation times, top-read fluorescence (RFUs; excitation $555 \mathrm{~nm}$ \& emission 585 $\mathrm{nm}$ ) was measured using SpectraMax M2 plate reader and SoftMax $\mathrm{Pro}^{\circ}$ v5.4 software (Molecular Devices, Sunnyvale, CA). Experiments were carried out in duplicate and data was acquired from at least 4 independent experiments. Data were expressed as a percentage of untreated cells. Graphing and calculation of $\mathrm{IC}_{50}$ values were accomplished using GraphPad Prism Software 4.0 (San Diego, CA).

\section{Statistical analysis}

For all experiments, samples were run in duplicate and data obtained were the result of 4-6 independent experiments, unless otherwise indicated. Data were graphed and analyzed using GraphPad Prism Software 4.0 using one-way ANOVA or two-way ANOVA and Tukey's post hoc test analysis or Bonferroni's correction where appropriate. Standard unpaired t-test also used when appropriate.

\section{Results}

\section{Long term EGF treatment results in a persistent expression of mesenchymal markers}

The ovarian cancer microenvironment is rich in activators of the
EGFR $[9,23]$, so we evaluated the effects of extended EGFR activation in ovarian cancer cells in vitro. After approximately 40 days of continuous treatment with 10nM EGF (long term EGF treatment), ovarian cancer (OVCA) 433 cells showed evidence of EMT as shown by reduced cellcell contacts and acquisition of a mesenchymal phenotype (Figure 1A). This change in phenotype of the cells was not fully reversible even after removal of EGF for approximately 7 days (removal). Epithelial (E-cadherin) and mesenchymal (N-cadherin and vimentin) proteins are common markers investigated in EMT. We observed no difference in E-cadherin protein from controls and long term EGF treated samples or long term EGF treated samples removed from EGF for 7 days (removal) (Figure 1B). Quantification of immunoblot intensities as well as mRNA expression levels for E-cadherin did not reveal a significant difference between groups (Figures 1C and 1D). We found no significant changes in other markers of EMT such as Snail, Slug or Twist in long term EGF treated samples (Supplementary Figure S1). Given that these transcription factors repress E-cadherin expression, this observation is consistent with the lack of change in E-cadherin after long term EGFR activation. In contrast, there was an increase in protein and mRNA expression levels for both $\mathrm{N}$-cadherin and vimentin following long term EGF treatment which persisted after ligand withdrawal. Representative western blots for $\mathrm{N}$-cadherin and vimentin are shown in Figure 1B. One way ANOVA followed by Tukey's post hoc of the data for quantified immunoblot intensities as well as mRNA expression levels of $\mathrm{N}$-cadherin revealed a significant increase in long term EGF treated samples as well as removal samples (Figures $1 \mathrm{E}$ and $1 \mathrm{~F}$ ). Similarly, data analysis of immunoblot intensities as well as mRNA expression levels of vimentin were also significantly increased in long term EGF treated samples as well as removal samples (Figure $1 \mathrm{G}, \mathrm{H}$ ). In accordance with these findings, our lab has previously shown that continuous activation of the EGFR by inducing expression of a constitutively active mutant receptor results in increased mesenchymal marker expression [24]. Furthermore, we found no significant differences in protein levels of other cadherins (H-cadherin, $\mathrm{P}$-cadherin and $\mathrm{OB}$-cadherin) with long term EGF (Supplementary Figure S2). There is evidence that cadherins mediate formation of multicellular aggregates $[8,25]$ so we next tested the impact of gene expression changes on MCA characteristics.

\section{Long term EGF results in compact multicellular aggregate formation (MCA) and increased cell spreading after adherence to collagen}

Initial experiments evaluating MCA formation after extended exposure to EGF demonstrated that in order to obtain significant increases in mesenchymal marker expression, cells had to be maintained in EGF for greater than 4 weeks (Figure 2A). To determine the functional significance of increased, and persistent, induction of mesenchymal markers we assessed multicellular aggregate formation since $\mathrm{N}$-cadherin and vimentin had previously been correlated in regulation of MCA compaction [8]. Cells were plated in Lipidure coated $U$ bottom plates and allowed to form MCAs overnight. Evaluation of MCA formation over weeks of EGF exposure revealed that MCA's became compact 1 week after mesenchymal markers were increased, approximately 5 weeks (Figure 2B); thus, all experiments were carried out after $\sim 40$ days in EGF. Following MCA formation, control, EGF and removal MCAs were transferred to collagen coated plates for an additional 24 hours of spreading (Figure 2C). While all MCAs were formed from 2500 cells originally plated and proliferation of EGF treated cells is not significantly changed after extended exposure to EGF (data not shown), the resulting long term EGF treated and removal MCAs were found to be significantly more compact than control MCAs (Figure 2D). One way ANOVA followed by posthoc analysis of the data showing measurement of the area of MCA 
A.
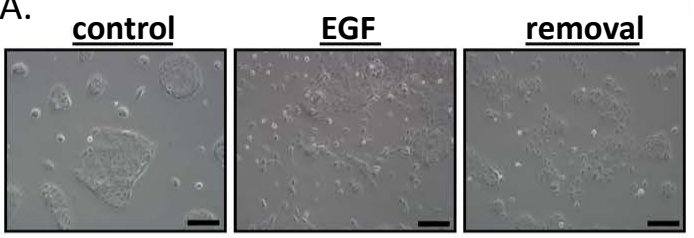

B.

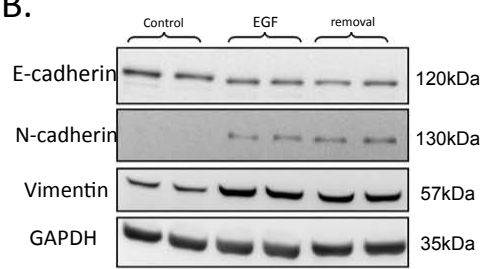

C.

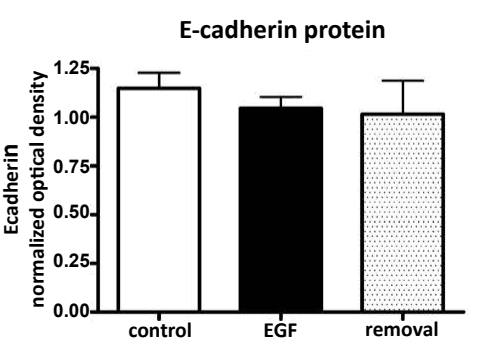

E.

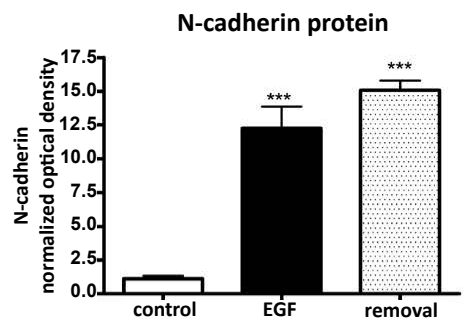

G.

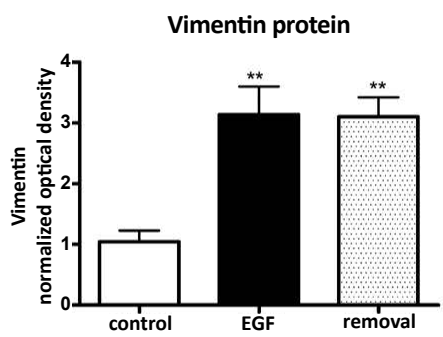

D.

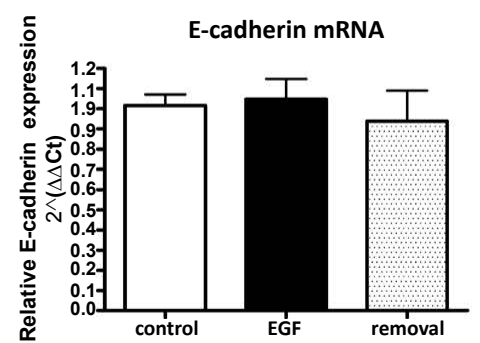

F.

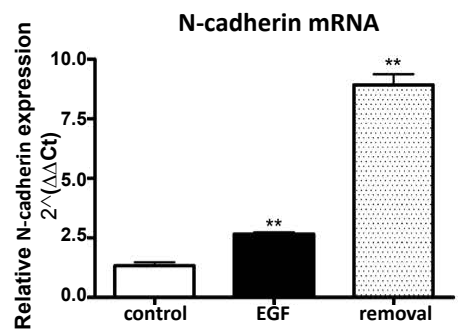

H.

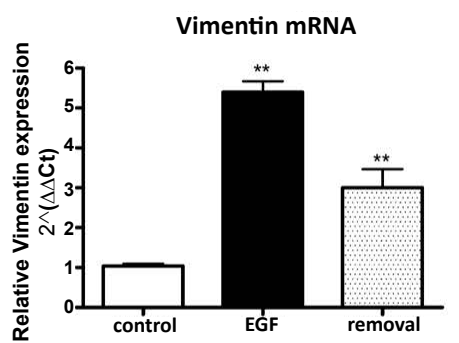

Figure 1: Long term incubation with Epidermal Growth Factor (EGF) causes persistent change in OVCA 433 cell morphology and induction of mesenchymal markers A) Representative phase images taken with 10X objective of OVCA 433 control, long term EGF-treated (EGF) and long term EGF treated cells in which the ligand was removed for at least 7 days (removal). Scale bar=200 $\mu \mathrm{m}$. B) Representative western blots of E-cadherin, N-cadherin and vimentin for control, EGF and 7 day removal (removal) samples with GAPDH as a loading control C) Quantification of normalized optical densities for E-cadherin in control, EGF and removal samples D) Relative E-cadherin mRNA expression. E) Quantification of normalized optical densities for N-cadherin in control, EGF and removal samples showed a significant increase in $\mathrm{N}$-cadherin protein in EGF and removal samples, $n=5,{ }^{* * *} p<0.001$. F) Relative $\mathrm{N}$-cadherin mRNA expression was also increased in EGF and removal samples, $n=5,{ }^{* *} p<0.01$. G) Quantification of normalized optical densities for vimentin in control, EGF and removal samples showed a significant increase in vimentin protein in EGF and removal samples, $n=5,{ }^{* *} p<0.01$. $H$ ) Relative vimentin mRNA expression was also increased in EGF and removal samples, $n=5,{ }^{* *} p<0.01$.

spreading revealed that EGF treated cells spread significantly further than control and removal cells (Figure 2E). However, removal cells also were significantly increased compared to control cells, showing that the increase in spreading conferred by long term EGFR activation is not a reversible phenotype. MCA formation was also evaluated in another ovarian cancer cell line that is known to have high levels of mesenchymal markers, SKOV3. SKOV3 cells show remarkably higher levels of both $\mathrm{N}$-cadherin and vimentin than long term EGF treated OVCA 433 cells; however, they show very little E-cadherin expression (Figure 3A). Indicative of their high mesenchymal marker expression, SKOV3 cells grown on a monolayer appear fibroblastic. Similar to the long term EGF treated cells, the SKOV3 MCAs are compact (Figure
3B). These findings suggest that mesenchymal marker expression is involved in MCA compaction.

\section{$\mathrm{N}$-cadherin mediates EGF-dependent changes in MCAs}

To test whether N-cadherin mediates MCA compaction and spreading, we used siRNA to knockdown N-cadherin in long term EGF treated cells; scramble siRNA was used as a negative control. The $\mathrm{N}$-cadherin (Ncad) siRNA reduced N-cadherin protein levels in EGF treated cells to a level that is comparable to passage control cells that were not treated with EGF (Figure 4A). One way ANOVA followed by Tukey's post hoc revealed a significant decrease in levels of N-cadherin in EGF treated cells with Ncad siRNA compared to EGF treated cells exposed to scramble siRNA or EGF treated cells that were not exposed 
A.

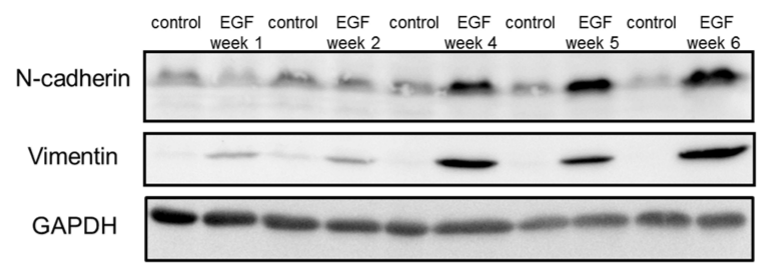

B.

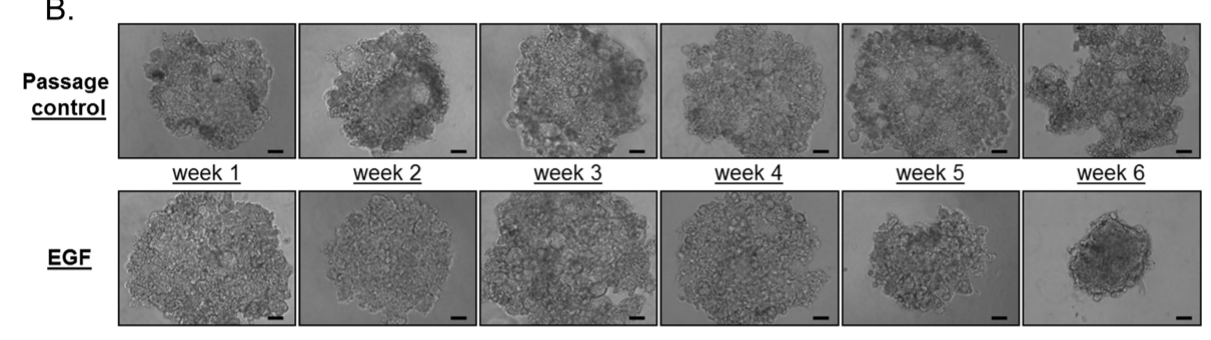

C.
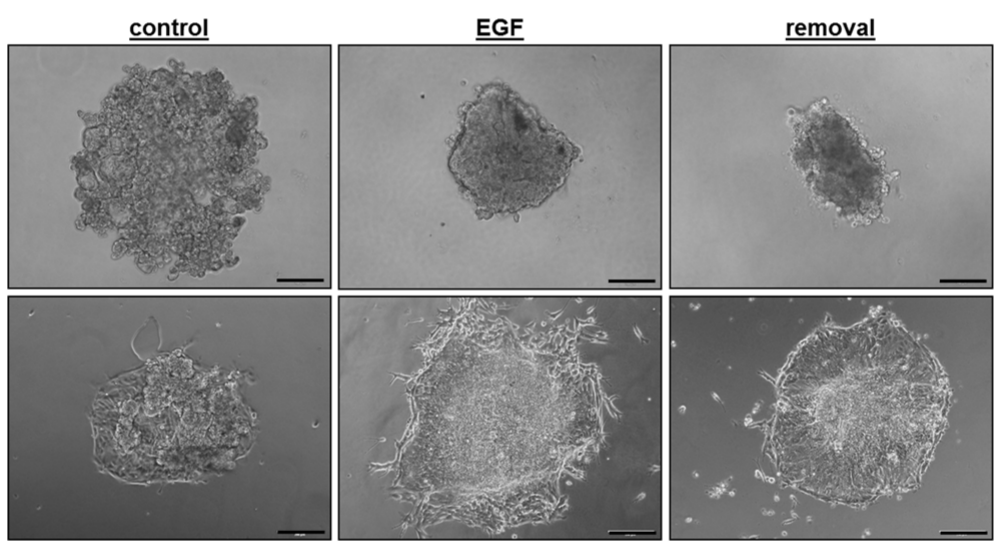

D.

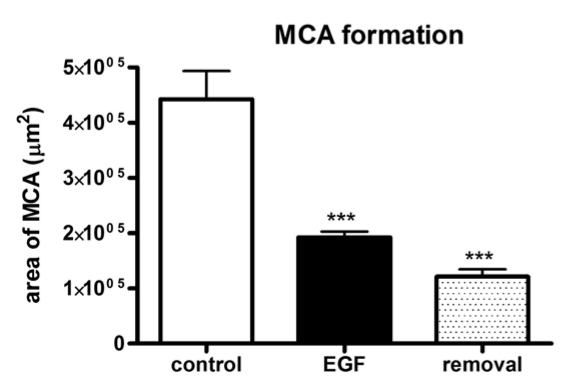

E.

\section{Spreading of MCA}

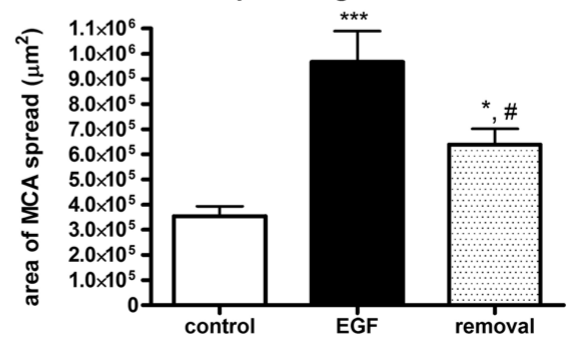

Figure 2: Long term EGF causes compact MCA formation and increased cell spreading. A) Representative western blot of N-cadherin and vimentin with GAPDH as a loading control over weeks of $10 \mathrm{nM}$ EGF treatment.B) Representative images of MCAs formed from passage controls and EGF treated cells over weeks of $10 \mathrm{nM} \mathrm{EGF}$ treatment. Scale bar=100 $\mu \mathrm{m}$ C) Representative 10X images of MCAs and MCA spreading on collagen from control, EGF and removal cells. Scale bar $=200 \mu \mathrm{m}$. D) Data showing area of the formed MCA was significantly, and irreversibly, decreased in EGF treated and removal cells compared to controls, $n=6,{ }^{* \star *} p<0.001$. E) Data of area of the spreading of MCAs on collagen showed a significant increase in EGF treated and removal cells compared to controls, $n=6,{ }^{* * *} p<0.001,{ }^{*} p<0.05$. Removal cell spreading was also significantly decreased compared to EGF, $\# p<0.05$.

to any siRNA treatment (Figure 4B). We were also able to significantly knockdown $\mathrm{N}$-cadherin protein in SKOV3 cells using siRNA (Figures $4 \mathrm{C}$ and $4 \mathrm{D})$. As shown in the images in Figure 5A, N-cadherin knock down largely reversed the MCA compaction observed in cells that received prolonged EGF treatment. The higher magnification images depict the defined edges present in the EGF treated scramble MCAs and the lack of those defined edges in both control and Ncad siRNA MCAs. One-way ANOVA followed by Tukey's post hoc test showed that the area of MCA compaction when N-cadherin protein was reduced in EGF treated cells was significantly increased compared to
EGF treated scramble siRNA MCAs (Figure 5B). Similarly, knockdown of N-cadherin in SKOV3 cells caused the MCAs to be less compact than control untreated SKOV3 MCAs or SKOV3 MCAs treated with scramble siRNA (Figures 5D and 5E). In addition, the area of MCA spreading was significantly increased in long term EGF treated cells exposed to Ncad siRNA compared to passage control MCAs (Figure 5C). These findings demonstrate that $\mathrm{N}$-cadherin knockdown largely reversed the long term EGF-induced MCA behavior (compaction and spreading) indicating that the persistent induction of N-cadherin following constitutive activation of the EGFR has a functional 


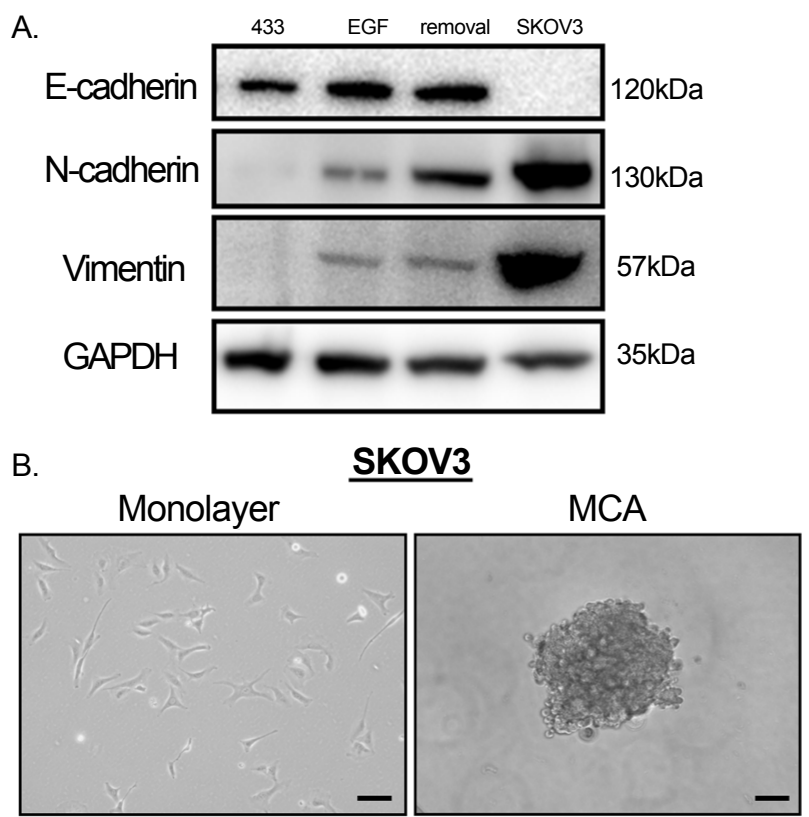

Figure 3: SKOV3 cells have robust expression of mesenchymal markers and show compact MCAs. A) Representative western blots of E-cadherin, N-cadherin and vimentin for control, EGF, removal and SKOV3 samples with GAPDH as a loading control. B) Representative $10 \mathrm{X}$ images of SKOV3 cells grown as a monolayer and as an MCA. Scale bar $=100 \mu \mathrm{m}$

A.

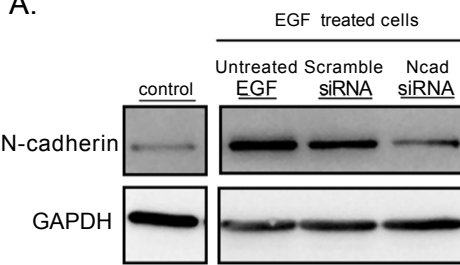

C.

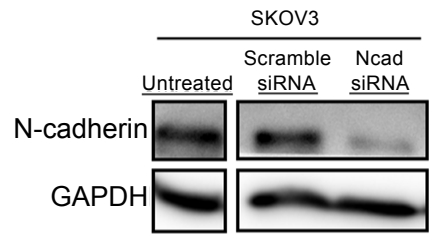

B.

$\mathrm{N}$-cadherin levels after siRNA

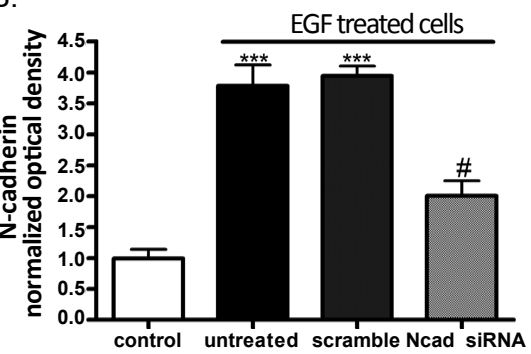

D. SKOV3 Ncadherin levels after siRNA

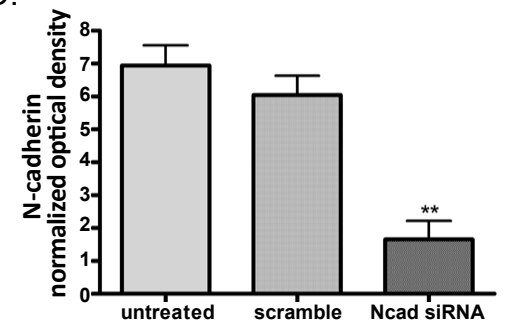

Figure 4: siRNA knockdown of N-cadherin in EGF treated cells and SKOV3 cells. A) Representative western blot showing N-cadherin expression in OVCA 433 passage control cells and EGF treated cells (untreated with siRNA) or treated with scramble siRNA or Ncad siRNA, GAPDH shown as a loading control. B) Normalized N-cadherin optical density in passage control and EGF treated cells without siRNA (untreated) or treated with scramble siRNA or Ncad siRNA, $n=5$. The significant increase in $\mathrm{N}$-cadherin in untreated and scramble (EGF treated cells) compared to passage control cells, ${ }^{* * *} p<0.001$ is blunted by treatment with Ncad siRNA, \#\#\#p $<0.001$ (significant decrease from untreated and scramble). C) Representative western blot showing N-cadherin expression in SKOV3 cells (untreated) and treated with scramble siRNA or Ncad siRNA, GAPDH shown as a loading control. D) Normalized N-cadherin optical density in SKOV3 cells untreated and treated with scramble siRNA or Ncad siRNA, $n=3$. Significant decrease in Ncad siRNA treated SKOV3s compared to untreated cells, ${ }^{* *} \mathrm{p}<0.01$.

significance in the formation of multicellular aggregates.

\section{Compact MCAs resulting from long term EGFR activation are more resistant to cisplatin treatment}

A described characteristic of MCAs is their resistance to chemotherapy $[2,8]$; thus, we aimed to test whether MCA compaction further modified drug resistance. Monolayers of cells and MCAs for control OVCA 433, EGF treated and removal cells as well as SKOV3 cells were treated with increasing doses of cisplatin. Figures 6A-D illustrate that MCAs are more resistant to cisplatin compared to their 
A.

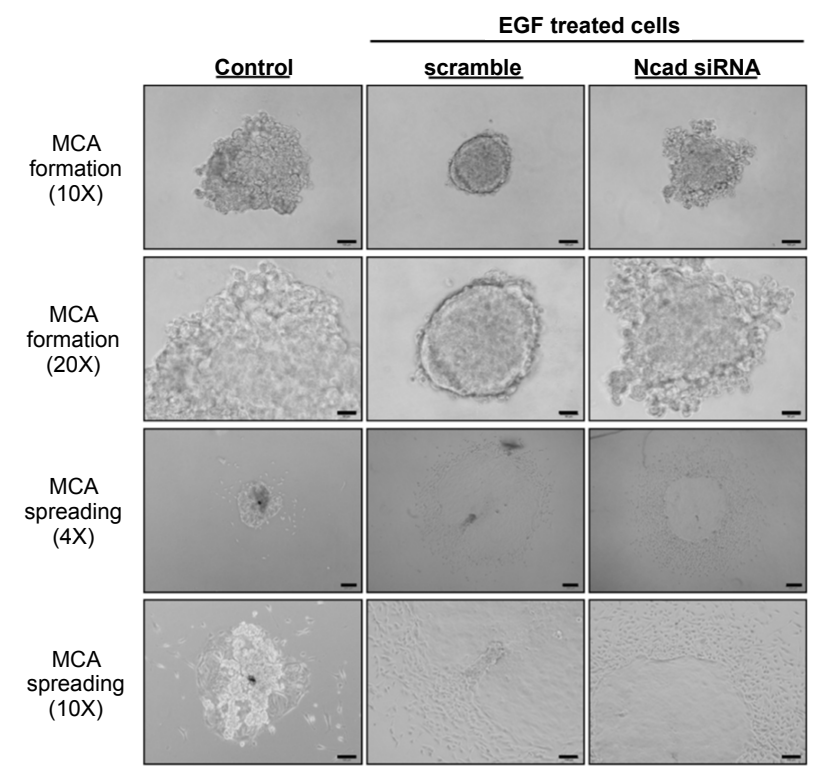

B.

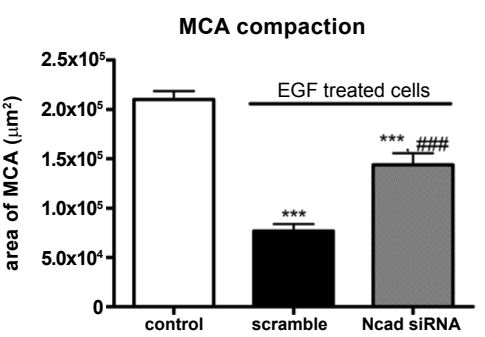

D.

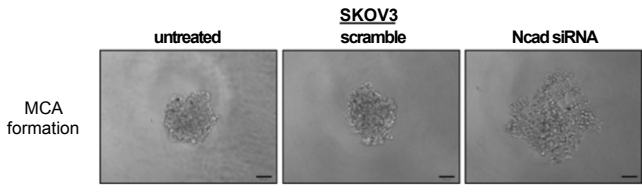

C.

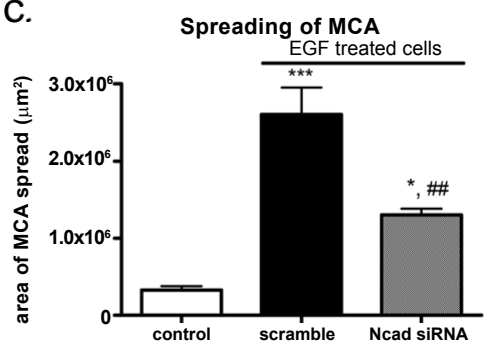

E.

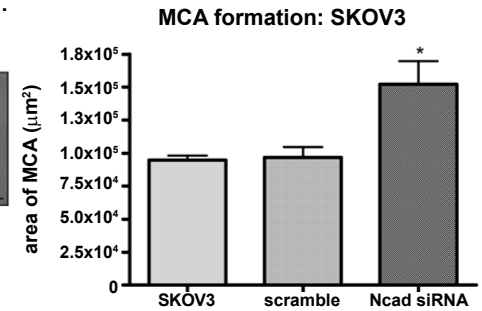

Figure 5: MCA formation and spreading after knockdown of N-cadherin in EGF treated cells. A) Representative images of passage control and EGF treated cells with scramble or Ncad siRNA. MCA formation images taken with $10 \mathrm{X}$ objective (scale bar=100 $\mu \mathrm{m})$ and $20 \mathrm{X}$ objective (scale bar $=50 \mu \mathrm{m})$. MCA spreading images taken with $4 \mathrm{X}$ objective (scale bar=200 $\mu \mathrm{m})$ and $10 \mathrm{X}$ objective $(\mathrm{scale}$ bar $=100 \mu \mathrm{m})$. B) Data showing area of the formed MCA was significantly decreased in EGF (scramble) cells compared to controls, $n=6,{ }^{* * *} p<0.001$. Knockdown of $\mathrm{N}$-cadherin (Ncad siRNA) exhibited a significantly decreased MCA formation area from passage control cells, ${ }^{* * *} \mathrm{p}<0.001$ but a significant increase in MCA formation area compared to EGF (scramble) cells, \#\#p<0.001. C) Data of area of the spreading of MCAs on collagen showed a significant increase in EGF (scramble) cells compared to controls, $n=6,{ }^{* * *} p<0.001$. Knockdown of $\mathrm{N}$-cadherin (Ncad siRNA) resulted in a significantly increased area of MCA spreading compared control cells, ${ }^{*} p<0.05$ but a significant decrease in MCA spreading area compared to EGF (scramble) cells, \#\#<0.01. D) Representative images SKOV3 MCAs untreated, scramble siRNA and Ncad siRNA treated taken with $10 X$ objective, scale bar=100 $\mu \mathrm{m}$. E) Data showing area of the formed SKOV3 MCAs, $n=4$. Knockdown of $\mathrm{N}$-cadherin (Ncad siRNA) exhibited a significantly increased area of MCA formation from untreated SKOV3 MCAs, ${ }^{*} p<0.05$.

respective monolayer cultures in all groups. Two-way ANOVA of the data in Figure 6A followed by post hoc analysis showed a significant difference between control monolayer viability and MCA viability at both 5 and $10 \mu \mathrm{M}$ cisplatin. $\mathrm{IC}_{50}$ values for control monolayer and MCAs were 19 and $35 \mu \mathrm{M}$ cisplatin, respectively. EGF treated monolayer and MCA showed a similar result (Figure 6B). Post hoc analysis showed a significant difference between EGF monolayer viability and MCA viability at 5,10,20,50 and $100 \mu \mathrm{M}$ cisplatin. IC $_{50}$ values for EGF monolayer and MCAs were 7 and $43 \mu \mathrm{M}$ cisplatin, respectively. Removal cell data for monolayer and MCA cultures also illustrated that MCAs are more resistant to chemotherapeutics (Figure 6C). Post hoc analysis showed a significant difference between removal monolayer viability and MCA viability at $5,10,20$ and $50 \mu \mathrm{M}$ cisplatin. IC $_{50}$ values for removal monolayer and MCAs were 9 and $47 \mu \mathrm{M}$ cisplatin, respectively. SKOV3 MCAs were also significantly different than monolayer cultures at $5,10,20,50,100 \mu \mathrm{M}$ cisplatin with $\mathrm{IC}_{50}$ values for monolayer and MCAs at 10.5 and $35 \mu \mathrm{M}$ cisplatin, respectively. Following knockdown of N-cadherin in long term EGF treated cells, MCAs became more sensitive to cisplatin compared to long term EGF treated MCAs given scramble siRNA. Two-way ANOVA followed by post hoc analysis showed that percent viability was significantly different at $100 \mu \mathrm{M}$ cisplatin. Highlighting the comparison between 
A.

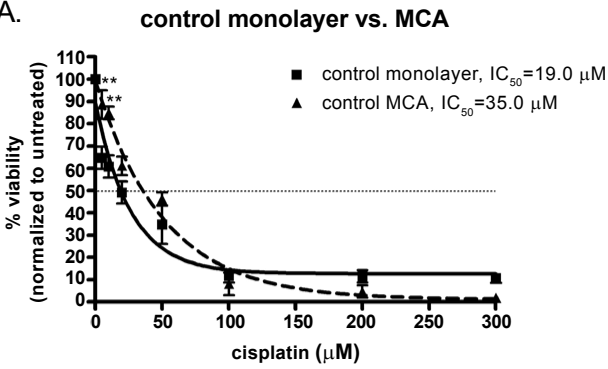

C.

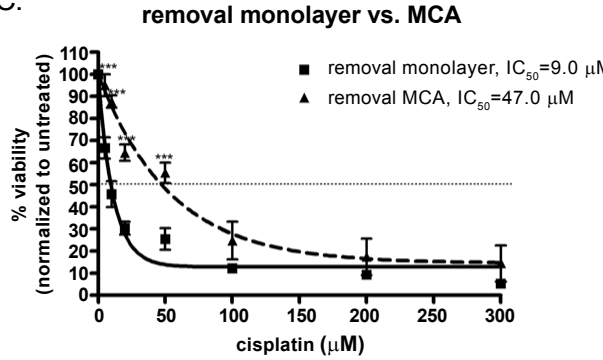

E.

MCA cisplatin viability after $\mathrm{N}$-cad knockdown

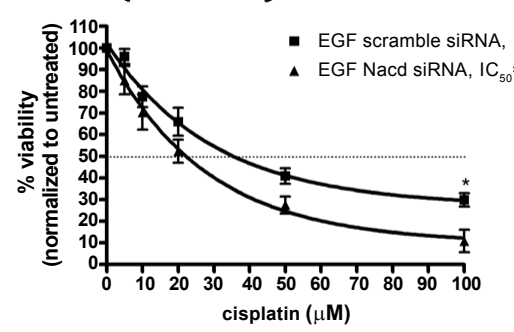

B.

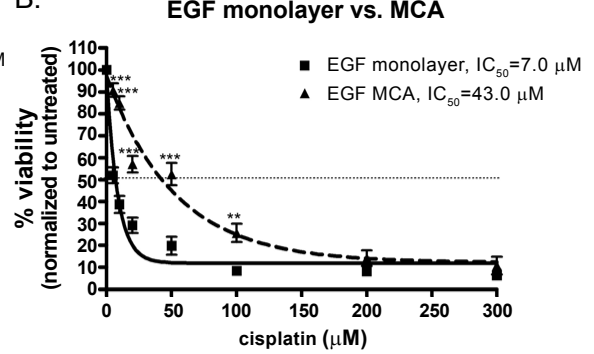

D.

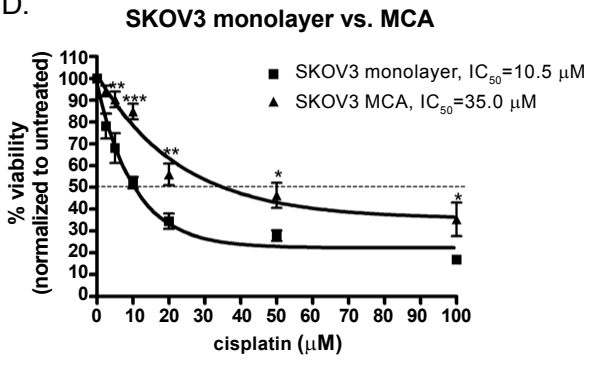

F.

MCA viability at $100 \mu \mathrm{M}$ cisplatin

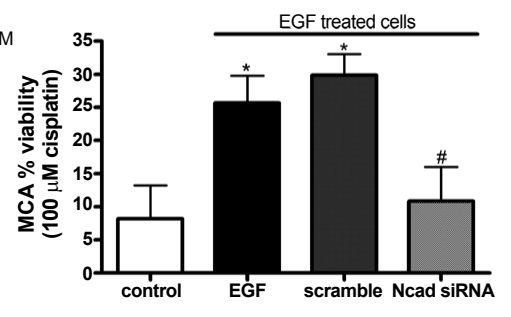

Figure 6: Compact MCAs demonstrate higher cell viability than monolayer cultures in response to 48hr of cisplatin and cells response to cisplatin is mediated, in part, by N-cadherin expression A) OVCA 433 passage control cell viability for monolayer (solid line) and MCAs (dashed line) in response to cisplatin. Data expressed as a percentage of untreated control cells, $n=4$. Two way ANOVA showed a significant overall main effect of monolayer vs. MCA [ $F(1,48)=9.711$, $p<0.01]$, a significant effect of cisplatin exposure $[F(7,48)=146.1, p<0.001]$ as well as a significant interaction $[F(7,48)=4.971, p<0.001]$. Significant differences in viability observed at 5 and $10 \mu \mathrm{M}$ cisplatin in control cells, ${ }^{* *} \mathrm{p}<0.01$. B) Long term EGF treated cells viability for monolayer (solid line) and MCAs (dashed line) in response to cisplatin. Data expressed as a percentage of untreated EGF cells, $n=4$. Two-way ANOVA of long term treated EGF cells data showed a significant overall main effect of monolayer vs. MCA $[F(1,48)=184.7, p<0.001]$, a significant effect of cisplatin exposure $[F(7,48)=205.6, p<0.001]$ as well as a significant interaction $[F(7,48)=14.6, p<0.001]$. Significant differences in viability observed at $5,10,20,50$ and $100 \mu M$ cisplatin in $E G F$ cells, ${ }^{* * *} p<0.001$, ${ }^{* *} p<0.01$. C) Removal cells viability for monolayer and MCAs in response to cisplatin. Data expressed as a percentage of untreated removal cells, $n=4$. Two-way ANOVA of removal cell data showed a significant overall main effect of monolayer vs. MCA $[F(1,48)=73.37, p<0.001]$, a significant effect of cisplatin exposure $[F(7,48)=96.12, p<0.001]$ as well as a significant interaction $[F(7,48)=4.873, p<0.001]$. Significant differences in viability observed at 5 , 10, 20 and $50 \mu \mathrm{M}$ cisplatin in removal cells, ${ }^{* \star *} \mathrm{p}<0.001$. D) SKOV3 cell viability for monolayer and MCAs in response to cisplatin. Data expressed as a percentage of untreated SKOV3 cells, $n=4$. Two-way ANOVA of SKOV3 cell data showed a significant overall main effect of monolayer vs. MCA [F $(1,42)=64.95, p<0.001]$, a significant effect of cisplatin exposure $[F(6,42)=82.17, p<0.001]$ as well as a significant interaction $[F(6,42)=2.592, p<0.05]$. Significant differences in viability observed at $5,10,20,50$ and $100 \mu \mathrm{M}$ cisplatin in SKOV3 cells, ${ }^{* * *} p<0.001,{ }^{* *} p<0.01,{ }^{*} p<0.05$. E) MCA cell viability in long term EGF treated cells with either scramble siRNA or Ncad siRNA in response to cisplatin. Data expressed as a percentage of untreated long term EGF cells, $n=5$. Two-way ANOVA of Ncad knockdown cell data showed a significant overall main effect of $\operatorname{siRNA}[F(1,48)=14.74, p<0.001]$, a significant effect of cisplatin exposure $[F(5,48)=84.80$, $p<0.001$ ] with no significant interaction. Significant differences in viability observed at $100 \mu \mathrm{M}$ cisplatin in Ncad knockdown cells, ${ }^{*} p<0.05$. F) MCA percent viability data for passage control, EGF treated cells without siRNA or with scramble siRNA or Ncad siRNA at $100 \mu M$ cisplatin, $n=4-5$. One way ANOVA followed by post hoc analysis showed a significant increase in long term EGF treated cells and long term EGF treated cells with scramble siRNA compared to passage control, ${ }^{*} p<0.05$. Long term EGF treated cells that were given Ncad siRNA showed a significant decrease from both EGF treated cells and long term EGF treated cells with scramble siRNA, \#p<0.05.

percent viability of control, EGF treated, EGF treated with scramble siRNA and EGF treated with Ncad siRNA MCAs at $100 \mu \mathrm{M}$ cisplatin, we find that both the EGF and EGF treated with scramble siRNA are significantly more resistant to cisplatin than control cells; however, when $\mathrm{N}$-cadherin is knocked down, there is a significant decrease in the percent viability compared to EGF treated MCAs (Figure 6F). This suggests that $\mathrm{N}$-cadherin, in part, mediates the observed response to cisplatin in long term EGF treated MCAs.

\section{Discussion}

A correlation between EGFR activation and poor patient prognosis is well documented in ovarian cancer; however, a clear mechanism for how EGFR activation confers poor prognosis has yet to be defined. This work makes a step towards elucidating the mechanistic link between EGFR activation and poor prognosis with $\mathrm{N}$-cadherin as a key regulator in this process. In this study, we analyzed the effects of constitutive EGFR activation on ovarian cancer cells in order to gain a better understanding of the molecular consequences resulting from the unique ovarian tumor microenvironment. We found that constitutive EGFR activation results in the acquisition of some EMT-like characteristics, such as a persistent increase in mesenchymal markers, specifically N-cadherin, as well as compact MCA formation. Compact 
MCAs were more resistant to cisplatin treatment and N-cadherin was found to be an important regulator of MCA compaction and also play a role in sensitivity to cisplatin. This work is particularly interesting as it provides mechanistic evidence that the molecular consequences to EGFR activation, specifically induction of $\mathrm{N}$-cadherin expression, can contribute to the development of resistance and can therefore promote tumor progression thereby leading to poor patient prognosis.

Activators of the EGFR are present at high levels in patient ascites and have also been shown to be produced by ovarian cancer cells $[9,26,27]$. Given that more than one third of patients present with ascites at diagnosis and almost all have ascites at recurrence [28] the current model evaluating long term or constitutive EGFR activation ( $\sim 40$ days) is a representation of the extended exposure to EGFR activators that the ovarian cancer cells experience within patient ascites. Acute EGFR activation (8-48 hrs) has previously been shown to be associated with EMT, downregulation of E-cadherin increased expression of transcription factors associated with EMT (Slug, Snail and Twist) $[16,29,30]$, but the previous studies did not establish whether these responses were durable or reversible. In the current studies, we found no changes in the levels of E-cadherin or E-cadherin transcription regulators (Slug, Snail, Twist) after long term EGF, although other markers of EMT such as vimentin and N-cadherin were upregulated suggesting that mechanisms and outcomes to short term versus persistent EGFR activation differ. Interestingly, continuous EGFR activation based on a mutation that confers constitutive activation and reduced capacity for internalization and degradation (EGFRvIII) [31], leads to upregulation of $\mathrm{N}$-cadherin and silencing of E-cadherin [24]. Within this model, we previously published results showing that that constitutive activation of mutant EGFRvIII results in (E-cadherin) $\mathrm{CDH} 1$ promoter methylation [32]. While even short term activation of the EGFR resulted in increased DNA methyltransferase activity and long term EGF treatment increased global DNA methylation, only the persistent activation conferred by the EGFRvIII mutant decreased E-cadherin expression via $\mathrm{CpG}$ island 1 promoter methylation [32]. We believe that differences in whether or not E-cadherin is downregulated in the constitutive EGFR activation models can be attributed accumulation of epigenetic alterations over extended EGFR activation that may not be evident at 40 days. However, differences in receptor recycling/degradation between the models may also be a contributing factor; EGF receptors typically recycle 3-5 times through the early endosome before they is selected for degradation [33], however EGFRvIII has a decreased capacity to internalize or degrade [31]. In addition, Wheelock et al. describe a scenario for cadherin switching in which E-cadherin expression does not change significantly, but N-cadherin is increased [17]. Since this was our observation, we believe this may be one example for which E-cadherin loss is not essential for the induction of some EMT-like characteristics, particularly mesenchymal marker induction. Furthermore, this suggests that even with continued E-cadherin expression, gain of $\mathrm{N}$-cadherin is sufficient to alter cell behavior and drug response as indicated by our studies. We focused on $\mathrm{N}$-cadherin in the current studies as we observed robust increases in its expression with long term EGF treatment both at the protein and mRNA level that significantly persisted even in the absence of EGF. This suggests a permanent change in the molecular programming within the ovarian cancer cell. Previous patient data concurs that $\mathrm{N}$-cadherin expression is an important marker in ovarian cancer metastasis as staining was found to be strong in primary tumors $(71 \%)$ and this immunoreactivity is maintained in $67 \%$ of paired metastatic lesions [3]. Patient data is corroborated by several in vitro studies correlating increased $\mathrm{N}$-cadherin expression with a more invasive phenotype $[17,34,35]$. Furthermore, while other studies have shown that additional cadherins such as P-cadherin and
OB-cadherin (or cadherin-11) are associated with acquisition of or maintenance of an invasive phenotype [35,36], we found no significant changes in either of these cadherins, nor any change in the type II cadherin $\mathrm{H}$-cadherin in long term EGF treated samples. Thus, since the literature clearly supported a role for $\mathrm{N}$-cadherin in tumor progression, our studies aimed to evaluate the functional consequence of increased $\mathrm{N}$-cadherin levels.

MCAs represent a 3D model in which to study tumor cells. Researchers use $3 \mathrm{D}$ models as opposed to $2 \mathrm{D}$ monolayers as it is a better representation of the tumor microenvironment and chemosensitivity in a $2 \mathrm{D}$ model may not accurately reflect the pathophysiological responses seen in patients $[37,38]$. It has been proposed that MCAs can gain invasive properties by undergoing EMT [39]. However, the expression of both E-cadherin and $\mathrm{N}$-cadherin, along with vimentin, have been implicated in MCA formation and compaction [8,39]. More specifically, N-cadherin levels have been shown to be important for MCA compaction in ovarian and breast cancer cells $[8,25]$. Thus, the observation of significantly more compact MCAs in long term EGF treated cells correlated well with our data showing persistent increase in $\mathrm{N}$-cadherin levels even though E-cadherin levels remained unchanged. As further evidence that mesenchymal markers are mediating this compact nature of MCAs, we used the SKOV 3 cell line which inherently does not express E-cadherin and has increased mesenchymal marker expression compared to OVCA 433. SKOV3 MCAs, as expected, are compact just as we observed in the long term EGF treated MCAs. In support of our results showing that extended EGFR activation confers compact MCA formation, Sodek et al. treated OVCAR-3 and SKOV3 cells with patient ascites, which is known to contain EGFR activators, and found that extended culture in ascites ( $\sim 10$ days) resulted in compact spheroid formation in both cell lines [39]. However in this previous study, the functional consequences to extended exposure to ascites and increased MCA compaction in the same cell line were not evaluated. The siRNA experiments described here illustrate that $\mathrm{N}$-cadherin is a key regulator of MCA compaction in long term EGF treated cells as well as SKOV3 MCAs. Interestingly, compact MCAs also showed significant increases in the attachment area (spreading) when allowed to adhere to collagen, but this response was significantly decreased when $\mathrm{N}$-cadherin was knocked down. Of note, we do not negate that other mesenchymal markers, such as vimentin which was upregulated in both long term EGF cells and SKOV3 cells, could also be regulators in these processes and further studies will evaluate the contribution of vimentin. Taken together, this suggests that compact MCAs, resulting from long term EGFR activation, have an increased capacity to re-attach and disaggregate quickly onto a conducive matrix, such as collagen. For the patient, this might suggest that compact MCAs may be better at infiltrating the mesothelia thereby leading to increased metastasis.

MCAs are believed to represent a key component of metastasis and platinum resistance, thus are attractive targets for therapy. The development of resistance to chemotherapeutic treatment is a major factor contributing to the low survival rate for ovarian cancer patients. Most patients are initially responsive to treatment, but a large majority of those patients will relapse due to acquired drug resistance [40]. Interestingly, studies have shown that levels of the EGFR ligand, HB-EGF, is significantly increased in ovarian cancer patient ascites [23] as well as in platinum resistant cells [41]. Cisplatin treatment itself is known to activate the EGFR and it is known that this activation is mediated by cleavage of HB-EGF [42]. Furthermore, hyperactivation of the EGFR in ovarian cancer cells is reported to be associated with cisplatin resistance [43]. Our studies looking at cell viability in response to cisplatin show that MCAs are significantly 
more resistant to cisplatin treatment compared to monolayers of cells in all groups evaluated, including SKOV3 cells. This observation that 3D models (MCAs) are more chemoresistant to their 2D counterparts (monolayer cultures) has also been documented by others [38]. The current work depicts a significant change in sensitivity to cisplatin using a 3D model (MCAs) within the same cell line after those cells had undergone extended exposure to an external growth factor, EGF, as well as reveals $\mathrm{N}$-cadherin as an important regulator of this change in sensitivity. When comparing control and long term EGF treated MCAs, we observed a significant increase in cell viability for compact (EGF treated) MCAs at higher doses $(100 \mu \mathrm{M})$ of cisplatin, which was largely reversed when $\mathrm{N}$-cadherin was silenced with siRNA. Thus, suggesting that $\mathrm{N}$-cadherin in addition to, or because of, its ability to regulate MCA compaction, it also plays a role in the cell's sensitivity to cisplatin. In support of this, $\mathrm{N}$-cadherin was previously shown to play a role in regulating rat surface epithelial cell apoptosis [44].

Although other EMT markers, Slug and Snail, have been associated with development of chemoresistance [43,45-47], these markers were not altered in our studies. Previous studies did not individually evaluate the contribution of $\mathrm{N}$-cadherin as a regulator of chemoresistance as several EMT markers were induced concurrently with upregulation of $\mathrm{N}$-cadherin. Our model of acquisition of some EMT-like characteristics, particularly $\mathrm{N}$-cadherin induction, and resistance to cisplatin provided a way to eliminate other EMT markers as potential regulators of this phenomenon and highlight $\mathrm{N}$-cadherin as a key regulator as shown by the $\mathrm{N}$-cadherin siRNA experiments which conferred more sensitivity to cisplatin. In support of preferentially evaluating $\mathrm{N}$-cadherin as a key regulator in platinum resistance, in vivo ovarian cancer studies have shown a significant negative association with high expression of $\mathrm{N}$-cadherin and progression-free survival $[3,48]$ and there is an increased trend of retention of $\mathrm{N}$-cadherin in metastatic lesions $[3,47]$ yet no significant relationship between $\mathrm{N}$-cadherin expression and ovarian cancer progression has been made within the field $[3,48]$. This work opens the door for further investigation linking $\mathrm{N}$-cadherin to the acquisition of drug resistance in ovarian cancer potentially as a biomarker for drug response.

Furthermore, recent studies have shown that EMT markers, including $\mathrm{N}$-cadherin, are upregulated in the formation of MCAs and that treatment with drugs targeting epigenetic mechanisms such as Trichostatin A (TSA) and 5-aza-2'-deoxycytidine (5-azaCdR) in combination with cisplatin suppressed EMT markers [6]. Additionally, TSA and 5-aza-CdR treatment increased sensitivity of the cells to cisplatin [6], thus supporting a role for EMT markers, like $\mathrm{N}$-cadherin, in cell sensitivity to cisplatin. It further suggests that epigenetic modifications may be mediating these changes in EMT markers and thus also influencing cell sensitivity to cisplatin. Taken together with previous work linking EGFR activation to increases in DNA methyltransferase (DNMT) activity and global methylation [32], it supports a role for the EGFR in the development of platinum resistance either by alterations to epigenetic mechanisms or persistent mesenchymal marker induction or both. We believe that the current work begins to illustrate an EGFR dependent mechanism, for which $\mathrm{N}$-cadherin is a key component, by which drug resistance and metastasis can arise in ovarian cancer patients. This is an important advancement towards delineating a clear mechanism linking EGFR activation to poor patient prognosis.

\section{Acknowledgements}

We thank Dr. Karen Cooper for technical assistance in setting up siRNA experiments and Ms. Michaela Granados and Mr. Raymond Lee for helping with immunoblotting. We also thank Dr. Sharon Stack for valuable discussion and insight early in development of this project and Dr. Angela Wandinger-Ness for thoughtful suggestions during the final stages manuscript preparation. Research reported in this publication was supported by RO1 CA109545, K12GM088021 and K01CA172591. The content is solely the responsibility of the authors and does not necessarily represent the official views of the National Institute of General Medical Sciences or the National Institutes of Health.

\section{References}

1. Siegel R, Naishadham D, Jemal A (2012) Cancer statistics, 2012. CA Cancer J Clin 62: 10-29.

2. Correa RJ, Peart T, Valdes YR, DiMattia GE, Shepherd TG (2012) Modulation of AKT activity is associated with reversible dormancy in ascites-derived epithelial ovarian cancer spheroids. Carcinogenesis 33: 49-58.

3. Hudson LG, Zeineldin R, Stack MS (2008) Phenotypic plasticity of neoplastic ovarian epithelium: unique cadherin profiles in tumor progression. ClinExp Metastasis 25: 643-655.

4. Davidowitz RA, Iwanicki MP, Brugge JS (2012) In vitro mesothelial clearance assay that models the early steps of ovarian cancer metastasis. J Vis Exp.

5. Sodek KL, Ringuette MJ, Brown TJ (2009) Compact spheroid formation by ovarian cancer cells is associated with contractile behavior and an invasive phenotype. Int J Cancer 124: 2060-2070.

6. Meng F, Sun G, Zhong M, Yu Y, Brewer MA (2013) Anticancer efficacy of cisplatin and trichostatin $\mathrm{A}$ or 5-aza-2'-deoxycytidine on ovarian cancer. $\mathrm{Br} \mathrm{J}$ Cancer 108: 579-586.

7. Lengyel E, Burdette JE, Kenny HA, Matei D, Pilrose J, et al. (2013) Epithelial ovarian cancer experimental models. Oncogene.

8. Shield K, Ackland ML, Ahmed N, Rice GE (2009) Multicellular spheroids in ovarian cancer metastases: Biology and pathology. GynecolOncol 113: 143148.

9. Hudson LG, Moss NM, Stack MS (2009) EGF-receptor regulation of matrix metalloproteinases in epithelial ovarian carcinoma. Future Oncol 5: 323-338.

10. Zeineldin R, Muller CY, Stack MS, Hudson LG (2010) Targeting the EGF receptor for ovarian cancer therapy. J Oncol 2010: 414676.

11. Liu Y, Burkhalter R, Symowicz J, Chaffin K, Ellerbroek S, et al. (2012) Lysophosphatidic Acid disrupts junctional integrity and epithelial cohesion in ovarian cancer cells. J Oncol 2012: 501492.

12. Wieduwilt MJ, Moasser MM (2008) The epidermal growth factor recepto family: biology driving targeted therapeutics. Cell Mol Life Sci 65: 1566-1584.

13. Normanno N, De Luca A, Bianco C, Strizzi L, Mancino M, et al. (2006) Epidermal growth factor receptor (EGFR) signaling in cancer. Gene 366: 2-16.

14. Siwak DR, Carey M, Hennessy BT, Nguyen CT, McGahren Murray MJ, et al (2010) Targeting the epidermal growth factor receptor in epithelial ovarian cancer: current knowledge and future challenges. J Oncol 2010: 568938.

15. Ahmed N, Maines-Bandiera S, Quinn MA, Unger WG, Dedhar S, et al. (2006) Molecular pathways regulating EGF-induced epithelio-mesenchymal transition in human ovarian surface epithelium. Am J Physiol Cell Physiol 290: C1532-1542.

16. Cheng JC, Auersperg N, Leung PC (2012) EGF-induced EMT and invasiveness in serous borderline ovarian tumor cells: a possible step in the transition to lowgrade serous carcinoma cells? PLoS One 7: e34071.

17. Wheelock MJ, Shintani Y, Maeda M, Fukumoto Y, Johnson KR (2008) Cadherin switching. J Cell Sci 121: 727-735.

18. Polyak K, Weinberg RA(2009) Transitions between epithelial and mesenchymal states: acquisition of malignant and stem cell traits. Nat Rev Cancer 9: 265-273.

19. Yilmaz M, Christofori G (2009) EMT, the cytoskeleton, and cancer cell invasion Cancer Metastasis Rev 28: 15-33.

20. Zeisberg M, Neilson EG (2009) Biomarkers for epithelial-mesenchymal transitions. J Clin Invest 119: 1429-1437.

21. Dumont N, Wilson MB, Crawford YG, Reynolds PA, Sigaroudinia M, et al. (2008) Sustained induction of epithelial to mesenchymal transition activates DNA methylation of genes silenced in basal-like breast cancers. ProcNatlAcadSci U S A 105: 14867-14872.

22. Livak KJ, Schmittgen TD (2001) Analysis of relative gene expression data using real-time quantitative PCR and the 2(-Delta DeltaC(T)) Method. Methods 25: 402-408.

23. Kasai N, Kobayashi K, Shioya S, Yoshikawa Y, Yotsumoto F, et al. (2012) Soluble heparin-binding EGF-like growth factor (HB-EGF) detected by newly 
developed immuno-PCR method is a clear-cut serological biomarker for ovarian cancer. Am J Transl Res 4: 415-421.

24. Zeineldin R, Rosenberg M, Ortega D, Buhr C, Chavez MG, et al. (2006) Mesenchymal transformation in epithelial ovarian tumor cells expressing epidermal growth factor receptor variant III. Mol Carcinog 45: 851-860.

25. Ivascu A, Kubbies M (2007) Diversity of cell-mediated adhesions in breast cancer spheroids. Int J Oncol 31: 1403-1413.

26. Fang X, Yu S, Bast RC, Liu S, Xu HJ, et al. (2004) Mechanisms for lysophosphatidic acid-induced cytokine production in ovarian cancer cells. J Biol Chem 279:9653-9661.

27. Hu YL, Tee MK, Goetzl EJ, Auersperg N, Mills GB, et al. (2001)Lysophosphatidic acid induction of vascular endothelial growth factor expression in human ovarian cancer cells. J Natl Cancer Inst93:762-768.

28. Ahmed N, Stenvers KL (2013) Getting to Know Ovarian Cancer Ascites: Opportunities for Targeted Therapy-Based Translational Research. Front Oncol 3. 256

29. Cowden Dahl KD, Symowicz J, Ning Y, Gutierrez E, Fishman DA, et al. (2008) Matrix metalloproteinase 9 is a mediator of epidermal growth factor-dependent e-cadherin loss in ovarian carcinoma cells. Cancer Res 68: 4606-4613.

30. Xu Z, Jiang Y, Steed H, Davidge S, Fu Y (2010) TGF ${ }^{2}$ and EGF synergistically induce a more invasive phenotype of epithelial ovarian cancer cells. BiochemBiophys Res Commun 401: 376-381.

31. Gan HK, Cvrljevic AN, Johns TG (2013) The epidermal growth factor receptor variant III (EGFRvIII): where wild things are altered. FEBS J 280: 5350-5370.

32. Samudio-Ruiz SL, Hudson LG (2012) Increased DNA methyltransferase activity and DNA methylation following Epidermal Growth Factor stimulation in ovarian cancer cells. Epigenetics 7: 216-224.

33. Clague MJ, Urbe S (2001) The interface of receptor trafficking and signalling. J Cell Sci 114: 3075-3081.

34. Stemmler MP (2008) Cadherins in development and cancer. Mol Biosyst 4 : 835-850.

35. Peinado H, Portillo F, Cano A (2004) Transcriptional regulation of cadherins during development and carcinogenesis. Int J DevBiol 48: 365-375.

36. Cheung LW, Leung PC, Wong AS (2010) Cadherin switching and activation of p120 catenin signaling are mediators of gonadotropin-releasing hormone to promote tumor cell migration and invasion in ovarian cancer. Oncogene 29 2427-2440.
37. Loessner D, Stok KS, Lutolf MP, Hutmacher DW, Clements JA, et al. (2010) Bioengineered 3D platform to explore cell-ECM interactions and drug resistance of epithelial ovarian cancer cells. Biomaterials 31: 8494-8506.

38. Lee JM, Mhawech-Fauceglia P, Lee N, Parsanian LC, Lin YG, et al. (2013) A three-dimensional microenvironment alters protein expression and chemosensitivity of epithelial ovarian cancer cells in vitro. Lab Invest 93: 528542 .

39. Sodek KL, Murphy KJ, Brown TJ, Ringuette MJ (2012) Cell-cell and cell-matrix dynamics in intraperitoneal cancer metastasis. Cancer Metastasis Rev 31: 397 414

40. Vaughan S, Coward JI, Bast RC Jr, Berchuck A, Berek JS, et al. (2011) Rethinking ovarian cancer: recommendations for improving outcomes. Nat Rev Cancer 11: 719-725

41. Tang XH, Deng S, Li M, Lu MS (2012) The anti-tumor effect of cross-reacting material 197, an inhibitor of heparin-binding EGF-like growth factor, in human resistant ovarian cancer. BiochemBiophys Res Commun 422: 676-680.

42. Yoshida T, Okamoto I, Iwasa T, Fukuoka M, Nakagawa K (2008) The anti-EGFR monoclonal antibody blocks cisplatin-induced activation of EGFR signaling mediated by HB-EGF. FEBS Lett 582: 4125-4130.

43. Yue P, Zhang X, Paladino D, Sengupta B, Ahmad S, et al. (2012) Hyperactive EGF receptor, Jaks and Stat3 signaling promote enhanced colony-forming ability, motility and migration of cisplatin-resistant ovarian cancer cells. Oncogene 31: 2309-2322.

44. Trolice MP, Pappalardo A, Peluso JJ (1997) Basic fibroblast growth facto and $\mathrm{N}$-cadherin maintain rat granulosa cell and ovarian surface epithelial cell viability by stimulating the tyrosine phosphorylation of the fibroblast growth factor receptors. Endocrinology 138: 107-113.

45. Chen X, Zhang J, Zhang Z, Li H, Cheng W, et al. (2013) Cancer stem cells epithelial-mesenchymal transition, and drug resistance in high-grade ovarian serous carcinoma. Hum Pathol 44: 2373-2384.

46. Haslehurst AM,Koti M, Dharsee M, Nuin P, Evans K et al. (2012) EMT transcription factors snail and slug directly contribute to cisplatin resistance in ovarian cancer. BMC Cancer 12: 91

47. Ahmed N, Abubaker K, Findlay J, Quinn M (2010) Epithelialmesenchyma transition and cancer stem cell-like phenotypes facilitate chemoresistance in recurrent ovarian cancer. Curr Cancer Drug Targets 10: 268-278.

48. Quattrocchi L, Green AR, Martin S, Durrant L, Deen S (2011) The cadherin switch in ovarian high-grade serous carcinoma is associated with disease progression. Virchows Arch 459: 21-29. 